\title{
Evaluación del gasto en la construcción de unidades de salud: ejemplo de participación comunitaria
}

\author{
José Antonio Tapia-Cruz, M en $C_{1}{ }^{(1)} 0$ swaldo Urdapilleta, MPP, (2) $O$ ctavio Gómez-Dantés, MSP, ${ }^{(3)}$ \\ Francisco Garrido-Latorre, Dr en C.(4)
}

\begin{abstract}
Tapia-Cruz JA, Urdapilleta O, Gómez-Dantés 0, Garrido-Latorre F. Evaluación del gasto en la construcción de unidades de salud: ejemplo de participación comunitaria. Salud Publica Mex 2003;45:483-491. El texto completo en inglés de este artículo está disponible en: http://www.insp.mx/salud/index.html
\end{abstract}

\section{Resumen}

Objetivo. D emostrar los beneficios económicos de la participación comunitaria en la construcción de unidades de salud. Material y métodos. Se realizó una evaluación de la eficiencia del gasto en la construcción de unidades de salud. Se comparó el gasto $/ \mathrm{m}^{2}$ y los tiempos de construcción de cada una de las 21 unidades auxiliares de salud y los 81 centros de salud rural dispersos edificados por el Programa de A poyo a los Servicios de Salud para Población A bierta a traves de tres modalidades de construcción: licitación pública nacional, invitación restringida y participación comunitaria. Este gasto también se comparó con el gasto monetario de otras unidades de salud similares edificadas a través de los programas normales de obra de otras instituciones. Se desarrolló un análisis univariado utilizando estadísticas no paramétricas y se diseñó un modelo matemático de ajuste a normal (bootstrapping) para analizar el gasto $/ \mathrm{m}^{2}$. Resultados La mediana de gasto $/ \mathrm{m}^{2}$ y de tiempos de entrega en las unidades auxiliares de salud y de gasto $/ \mathrm{m}^{2}$ para los centros de salud rural dispersos fue menor cuando se empleó la participación comunitaria. Los gastos $/ \mathrm{m}^{2}$ de las unidades construidas por el Programa de Apoyo a los Servicios de Salud para Población A bierta, sobre to do con participación comunitaria, fueron considerablemente más eficientes que los reportados por las obras de otros programas de construcción. Conclusiones El uso de la par-

\author{
Tapia-Cruz JA, Urdapilleta O, \\ Gómez-Dantés 0, Garrido-Latorre F. \\ Expenditure evaluation of the construction of health units: \\ Example of community participation. \\ Salud Publica Mex 2003;45:483-491. \\ The English version of this paper \\ is available at: http://www.insp.mx/salud/index.html
}

\begin{abstract}
A bstrac
Objective. Demonstrate the economic benefits that community participation may render in the construction of health units. Materials and Methods. The purpose of this study was to analyze the efficiency in the construction of 21 auxiliary health units and 81 rural health built through the Program to Support the Development of Health Services for the N on-Insured Population (PASSPA) through three different construction models (national public bidding, restricted invitation and community participation). Comparisons were also made with the units built through regular construction procedures of the Ministry of Health and other institutions. To evaluate the expenditure $/ \mathrm{m}^{2}$, a univariate analysis using non-parametric statistics and a mathematical model of bootstrapping were used. Results. The median expenditure $/ \mathrm{m}^{2}$ and the delivery times for auxiliary health units, and the median expenditure $/ \mathrm{m}^{2}$ for rural health units were smaller when using community participation. The expenditure/ $\mathrm{m}^{2}$ of tho se units built thro ugh PASSPA was considerably lower than that of health units built through regular construction procedures. Conclusions The use of community participation in the construction of auxiliary health units and rural health units may impact positively the resource investment and the delivery times when compared with units built through national public biddings and restricted invitations. The possibility of using community parti-
\end{abstract}

(1) Subdirección de Evaluación de Servicios N o Personales de Salud; D irección General de Evaluación del D esempeño. Secretaría de Salud (SSA). México, DF, México.

(2) Universidad de Carolina del N orte, Estados Unidos de América.

(3) Dirección General de Evaluación del Desempeño. SSA. México, DF, México.

(4) Dirección de Evaluación de Servicios de Salud; D irección General de Evaluación del Desempeño, SSA. México, DF, México.

Fecha de recibido: 28 de junio de 2002 - Fecha de aceptado: 14 de agosto de 2003 Solicitud de sobretiros: José Antonio Tapia Cruz. Dirección General de Evaluación del Desempeño. Secretaría de Salud. Reforma 450, Piso 12, Colonia Juárez, 06600 México, DF, México. Correo electrónico: jatapia@salud.gob.mx 
ticipación comunitaria en la construcción de unidades auxiliares de salud y todos los centros de salud rural dispersos permite disminuir considerablemente el monto de recursos invertidos por $\mathrm{m}^{2}$ y mejorar los tiempos de construcción en relación con la licitación pública nacional y la invitación restringida. La construcción de unidades de salud bajo las condiciones generadas por el Programa de A poyo a los Servicios de Salud para la Población Abierta permitiría mejorar los gastos $/ \mathrm{m}^{2}$ en relación con otros programas de construcción. D ebe valorarse la viabilidad de emplear participación comunitaria en otras actividades normales de los servicios de salud. El texto completo en inglés de este artículo está disponible en:http://www.insp.mx/salud/index.html

Palabras clave: evaluación del gasto; eficiencia; participación comunitaria; gasto de construcción; tiempos de construcción; modalidades de construcción; México cipation in other health activities (super vision, maintenance) should be evaluated.The English version of this paper is available at: http://www.insp.mx/salud/index.html

Key words: expenditure evaluation; efficiency; community participation; construction expenditure; construction times; construction models; Mexico a participación comunitaria (PC) no es nueva. Lo L que sí puede considerarse novedoso es el esfuerzo por institucionalizarla en campos tan diversos como la educación, la industria, la salud, la agricultura y la política. $^{1}$

La PC ha tenido un desarrollo histórico amplio. Sin embargo, es en los años 60 y 70 cuando adquiere un gran impulso y se constituye en un elemento central de las políticas nacionales y de las agencias internacionales. En América Latina destaca el Programa de Unidades Móviles para Areas Rurales, financiado por la Alianza para el Progreso a principios de la década de los sesenta. En 1977, la Organización Mundial de la Salud (OMS) y el Fondo de las Naciones Unidas para la Infancia (UNICEF) publican los resultados de un estudio sobre participación comunitaria en programas de salud que incluía experiencias de México y Costa Rica. $^{2}$ En 1978, durante la Conferencia de Atención Primaria a la Salud, en Alma Ata, ${ }^{3}$ el vínculo entre participación comunitaria y atención a la salud se convirtió en la estrategia central para dar cumplimiento al objetivo de Salud para Todos en el Año 2000, establecido por la OMS en $1978 .^{4}$

Al término "participación comunitaria" se le han otorgado significados muy diversos. Según la OMS, ${ }^{5}$ se trata de:

El proceso en virtud del cual los individuos y las familias se responsabilizan por su salud y bienestar, y por la salud y bienestar de la colectividad, y mejoran su capacidad de contribuir a su desarrollo económico y al desarrollo comunitario.

Zakus, $^{6}$ por su parte, la define como:
El proceso a través del cual los miembros de una comunidad, individual o colectivamente, y con variaciones en el nivel de compromiso: a) desarrollan la capacidad para evaluar sus necesidades de salud y sus problemas; b) generan planes y los implantan buscando soluciones; c) crean y mantienen organizaciones que soportan estas acciones, y d) evalúan los efectos y llevan a cabo ajustes en los objetivos y programas en curso.

Como puede desprenderse de estas definiciones, las acciones comunitarias tienen como ventajas principales el permitir la identificación de las necesidades más sentidas, facilitar la instrumentación de posibles soluciones, atendiendo a las condiciones culturales locales, e incrementar las posibilidades de sustentar los proyectos, que son de mucha más fácil apropiación. La PC, además, puede constituirse en una función de producción alternativa en la generación y/o administración de bienes y servicios.

Según algunos autores la característica central de la PC es la participación en la toma de decisiones. En la literatura sobre el tema, de hecho, se cuenta con escalas de participación comunitaria que toman como criterio único para medir el grado de participación precisamente la toma de decisiones. Klein, ${ }^{7}$ por ejemplo, construyó una escala que ubica en el grado 1 el simple proceso de "información a la comunidad" y en el grado 5 el "veto", al que define como "la intervención en la toma de decisiones con la capacidad para bloquearlas una vez tomadas". La escala de Dachler y Wilpert $^{8}$ ubica en el extremo inferior la "no participación", a la que definen como "la no información a la comunidad", y ubica en el extremo superior al "control total de todos los involucrados en el proceso". Rifkin y 
colaboradores, ${ }^{9}$ por su parte, proponen una metodología para medir la participación comunitaria atendiendo no sólo a la participación en la toma de decisiones sino también a la participación en la evaluación de necesidades, el tipo de liderazgo, y la participación en la instrumentación de los proyectos o programas y en la movilización de recursos.

Así, dependiendo del énfasis en alguna o algunas de sus características, la PC puede entenderse como "fin" o como "medio" para cumplir con determinados objetivos. Cuando se le asume como "fin" se destaca la participación como mecanismo liberador que incrementa las posibilidades de las comunidades de asumir el control de sus vidas y destinos. ${ }^{10}$ Cuando se le mira como "medio", ya sea técnico o político, se privilegian los beneficios -económicos, en salud u otrosque pudiera generar, independientemente del fortalecimiento comunitario al que pudiera asociarse. Ugalde ${ }^{11}$ critica este último tipo de énfasis al señalar que en América Latina, en el campo de la salud, la PC ha servido de mecanismo legitimador de políticas que los organismos internacionales le han impuesto a los países de la región y que han empobrecido a la población más necesitada.

La evaluación de la PC tiende a facilitarse cuando se le asume como medio, con objetivos bien definidos y medibles, y se dificulta cuando se le trata exclusivamente como fin.

A pesar del desarrollo de metodologías para el estudio y medición de la PC, según Zakus, ${ }^{6}$ existe muy poca información empírica en la literatura sobre salud que documente las bondades o limitaciones de dicha participación. En general, se ha hecho énfasis en sus efectos positivos atendiendo exclusivamente a experiencias personales y a consideraciones teóricas.

Este trabajo -en el que se asume a la PC como un medio- se justifica porque aporta información empírica sobre la eficiencia del gasto monetario asociado a la PC en el campo de la salud y, en esa medida, apoya los procesos de toma de decisiones y de formulación de políticas en este sector. Aquí se documenta la participación de diversas comunidades en la construcción de unidades de salud de primer nivel de atención en México en el contexto de un programa de ampliación de cobertura de la Secretaría de Salud que se implantó entre 1991 y 1996 -Programa de Apoyo a los Servicios de Salud para Población Abierta (PASSPA)- en los estados más pobres del país (Chiapas, Guerrero, Hidalgo y Oaxaca). Su objetivo es demostrar, a través de la evaluación del gasto en la construcción de unidades de salud por $\mathrm{m}^{2}$ y a través de los tiempos de entrega, la eficiencia atribuible a la PC en relación con otras modalidades de construcción utilizadas en el PASSPA, y comparar los gastos de construcción de estas unida- des, principalmente de las construidas a través de la PC, con los reportados por los programas normales de obra de otras instituciones de salud (Instituto Mexicano del Seguro Social -IMSS-) y de desarrollo social (Programa Nacional de Solidaridad -Pronasol-).

Cabe señalar que la evaluación del gasto es un proceso técnico, administrativo y contable a través del cual se verifican y comparan los resultados con las metas y objetivos después de efectuar los gastos corrientes y de inversión. Cierto es que un análisis económico hubiera permitido obtener información más precisa sobre los costos y los beneficios de las diferentes modalidades de construcción analizadas. Sin embargo, diversas limitaciones de infraestructura, recursos y tiempo impidieron realizar un análisis de este tipo. A pesar de ello, en este trabajo se analiza la eficiencia en la construcción de unidades, es decir el logro de los objetivos planteados utilizando la menor cantidad de recursos posibles. ${ }^{12}$

El PASSPA tuvo como objetivo ampliar la cobertura de servicios de salud en los estados con mayores índices de marginación y las condiciones de salud menos favorables, y sustentó su operación en dos componentes: 1) componente de desarrollo de los servicios de salud y 2) componente de desarrollo institucional. El primero es el que regula las acciones de obra civil con base en la Ley de Adquisiciones y Obras Públicas (LAOP). ${ }^{13}$

Entre los dos componentes se distribuyeron los recursos presupuestales, y se canalizó un mayor volumen de ellos al componente de desarrollo de servicios. Sin embargo, no existió una directriz explícita para determinar la efectividad del uso de estos recursos. No obstante, los resultados obtenidos ofrecen la posibilidad de abordar este aspecto como tema de investigación.

Es importante mencionar que los procesos de construcción y rehabilitación de la infraestructura en el sector público mexicano normalmente se realizan a través de compañías constructoras privadas, ya que la LAOP restringe las modalidades de edificación a la licitación pública nacional (LPN), la invitación restringida (IR) y la asignación directa (AD).* Estas tres mo-

\footnotetext{
* Según la Ley de Adquisiciones y Obras Públicas de México, las obras públicas y adquisiciones con un costo menor a 45 mil pesos pueden realizarse a través de "asignación directa". Las obras y adquisiciones que sobrepasan este monto y hasta un máximo de 340 mil pesos se adjudican a través de "invitación restringida", en la cual se invita a por lo menos tres contratistas para que concursen por la obra. Las obras que sobrepasan los 340 mil pesos requieren de una "licitación pública nacional", que supone la invitación a todos los contratistas dentro del territorio nacional para que presenten sus propuestas.
} 
dalidades fueron las que se utilizaron al inicio del PASSPA. Sin embargo, eventualmente (1993) se autorizó la PC como mecanismo alternativo de construcción.

La PC en este estudio supone el involucramiento de la población en la construcción de unidades de salud a través de la conformación de comités que tenían la responsabilidad de: a) facilitar el terreno; b) realizar las cotizaciones; c) comprar los materiales; d) ejecutar las obras en los plazos programados, y e) informar sobre el avance físico de la obra y el ejercicio de los recursos financieros. Es importante señalar que la edificación de estas obras ofreció a los individuos de la comunidad la posibilidad de recibir un pago por su trabajo sobre la base de un salario mínimo por día laborado, lo que representó un beneficio directo adicional para la economía de la región. Estos comités contaron con el apoyo de las autoridades municipales y de las autoridades locales de los servicios de salud.

Otro aspecto que es necesario resaltar es el de la calidad de las obras. Dentro del PASSPA se aseguró, a través de la constitución de Direcciones de Obra e independientemente del tipo de modalidad de construcción, que las obras cumplieran con criterios de calidad previamente definidos -tanto de materiales como de las edificaciones-. Esto permitió homogeneizar criterios para la elaboración de los expedientes técnicos de cada proyecto de construcción, los cuales incluían especificaciones sobre las dimensiones de las unidades, tipos de materiales a emplearse y características de los tiempos de construcción. Además, estas Direcciones tenían la responsabilidad de supervisar cada una de las obras y, en los casos en los que se utilizó la PC, ofrecer el apoyo técnico necesario para su adecuada ejecución.

\section{Material y métodos}

Se desarrolló una evaluación de la eficiencia del gasto, que se definió como aquella función de producción que genera los mejores resultados en términos del uso de los recursos financieros para la construcción de unidades de salud y tiempos de ejecución. ${ }^{14}$

Para este estudio se seleccionaron 102 unidades de las 113 construidas entre 1991 y 1996 por el PASSPA en los estados de Guerrero e Hidalgo, que fueron los estados en los que se construyó obra con PC. El criterio de inclusión fue la existencia de por lo menos un tipo de unidad edificada a través de cada una de las tres modalidades de construcción utilizadas por el programa: LPN, IR y PC. Los tipos de unidades construidas fueron unidades auxiliares de salud (UAS), centros de salud rurales dispersos (CSRD), centros de salud rurales concentrados (CSRC) y centros de salud urba- nos (CSU). El criterio de inclusión sólo se cumplió para los CSRD y las UAS, por lo que se dejaron fuera seis CSU construidos en Guerrero y cinco CSRC, tres construidos en Hidalgo y dos en Guerrero. Según el Modelo de Atención a la Salud para Población Abierta (MASPA), ${ }^{15}$ las UAS se ubican en localidades de 500 a 1000 habitantes -normalmente de difícil acceso-. Su infraestructura se compone de un consultorio y una sala de espera, y se operan con un médico general o un pasante de la carrera de medicina. Los CSRD atienden a una población de 1000 a 2500 habitantes, y para ello cuentan con uno a tres consultorios de medicina general, atendidos cada uno por un médico general o un pasante de la carrera de medicina, una enfermera y un promotor de salud.

Las variables dependientes fueron el gasto $/ \mathrm{m}^{2}$ en pesos y tiempo de construcción de la unidad en días. Los costos unitarios se deflactaron utilizando el Indice Nacional de Precios del Sector Secundario (construcción), tomando como base el año 1990. Los tiempos de construcción se evaluaron tomando como referencia los plazos normados para el programa de obra civil del PASSPA, que fueron de 150 días para unidades de fácil acceso, 188 días para unidades de acceso intermedio y 225 días para unidades de difícil acceso. El grado de accesibilidad se incluyó para eliminar distorsiones relacionadas con la ubicación geográfica de las unidades o centros. La clasificación utilizada en este último caso tomó como criterios el tiempo de traslado y el tipo de vías de comunicación que existían entre el centro de distribución de insumos y el área de construcción. Se definieron tres categorías: a) zona de fácil acceso, que implica un tiempo de traslado de hasta dos horas en carretera a los centros de abasto; b) zona de acceso regular, con un tiempo de traslado de hasta cuatro horas en carretera o una hora y media en camino de terracería, y c) zona de difícil acceso, que comprende un tiempo de traslado de más de una hora y media en camino de terracería o más de cuatro horas en carretera.

Se compararon los costos unitarios y los tiempos de construcción de cada una de las unidades PASSPA de las diversas modalidades de construcción, y posteriormente se hicieron comparaciones similares con las unidades construidas a través de los programas regulares de construcción de la SSA, IMSS y del Pronasol.

Para cada una de las características se hizo un análisis univariado que se resumió utilizando medidas de tendencia central, de dispersión y porcentajes, según el caso. Debido a que las observaciones para las variables gasto $/ \mathrm{m}^{2}$ y tiempo de ejecución presentaron una distribución sesgada, se optó por utilizar estadísticas no paramétricas: la prueba de Kruskal-Wallis para 
comparar las medianas del gasto $/ \mathrm{m}^{2}$ con las variables de más de dos categorías y la prueba U de MannWhitney para las variables dicotómicas. Ambas pruebas se emplean para analizar variables independientes, como es el caso de las observaciones obtenidas en este estudio.

Finalmente, se llevó a cabo un análisis matemático de ajuste a normal de bootstrapping para analizar el comportamiento del gasto $/ \mathrm{m}^{2}$. La unidad de comparación en el análisis fue el gasto $/ \mathrm{m}^{2}$ de las UAS construidas con PC en localidades de fácil acceso en el estado de Hidalgo, que en teoría, por su tamaño, complejidad y ubicación geográfica, deberían tener el menor costo. El análisis se realizó con el paquete estadístico STATA versión 6.0 y 7.0.

\section{Resultados}

En los dos estados involucrados en este estudio el PASSPA construyó 113 unidades. Las unidades objeto de estudio, de acuerdo con el criterio de inclusión, fueron 102, de las cuales 33 corresponden a Hidalgo y 69 a Guerrero. De éstas, 46 se edificaron en zonas de difícil acceso, 25 en zonas de acceso intermedio y 31 en zonas de fácil acceso. La modalidad administrativa utilizada para realizar las obras fue principalmente la LPN (50), seguida por la construcción a través de PC (34). La inversión total efectuada por el programa de construcción en los dos estados fue de aproximadamente 14.2 millones de pesos reales, que representaron $57 \%$ de los recursos presupuestarios totales empleados en obra civil.

Por lo que se refiere a las 21 UAS -todas construidas en Hidalgo-, cuatro se construyeron a través de LPN, nueve a través de IR y ocho con PC. La mayoría (15) se ubicó en zonas de difícil acceso.

El gasto total de construcción de estas unidades fluctuó entre \$93880 y \$157 752. La mediana del gasto $/ \mathrm{m}^{2}$ ascendió a $\$ 1017$. Como puede observarse en el cuadro I, la mediana de gasto $/ \mathrm{m}^{2}$ de construcción fue más baja cuando la obra se construyó con PC. Si el análisis se desagrega por tipo de acceso se observan resultados similares. En áreas de acceso intermedio se construyeron cuatro UAS, cuyos gastos totales fluctuaron entre $\$ 93880$ y $\$ 145120$. Las dos UAS construidas con PC en zonas de fácil acceso presentaron una mediana de $\$ 880.5$ por $\mathrm{m}^{2}$. En las zonas de difícil acceso, el gasto $/ \mathrm{m}^{2}$ fluctuó entre $\$ 896$ y \$1 252. Sólo siete de las unidades construidas en estas zonas presentaron gastos/ $\mathrm{m}^{2}$ menores a la mediana; cuatro se construyeron con PC. El tiempo de conclusión se consideró aceptable en sólo dos unidades, de acuerdo con los parámetros de construcción por tipo de acceso. La me-

\section{Cuadro I \\ GaSTO/M $^{2}$ Y TIEMPOS DE CONSTRUCCIÓN EN UNIDADES AUXILIARES DE SALUD, SEGÚN MODALIDAD de Construcción Y tipo de acceso. Programa de Apoyo a los Servicios de Salud para Población Abierta. Hidalgo, México, 2000}

Gasto/m² Tiempos de construcción* Variable Unidades Mediana Mínimo Máximo Mediana Mínimo Máximo

Modalidad

\begin{tabular}{lrrrrrrr}
$\mathrm{PC}^{\ddagger}$ & 8 & 897.3 & 853.4 & 927.7 & 245.6 & 113.0 & 431.0 \\
\hline $\mathrm{LPN}^{\S}$ & 4 & 1138.8 & 1021.1 & 1251.7 & 626.8 & 338.0 & 1001.0 \\
\hline $\mathrm{IR}^{\#}$ & 9 & 1071.2 & 896.3 & 1186.3 & 596.2 & 203.0 & 928.0
\end{tabular}

Acceso

\begin{tabular}{crrrrrrr} 
Fácil & 2 & 880.5 & 853.4 & 907.5 & 174.0 & 168.0 & 180.0 \\
\hline Regular & 4 & 981.5 & 853.4 & 1151.7 & 365.0 & 113.0 & 775.0 \\
\hline Difícil & 15 & 1045.8 & 896.3 & 1251.7 & 535.0 & 203.0 & 1001.0 \\
& & & & & & & \\
Global & 21 & 1017.8 & 853.4 & 1251.7 & 468.5 & 113.0 & 1001.0
\end{tabular}

* Gasto/m² en pesos y tiempos de construcción en días

₹ Participación comunitaria

$\S$ Licitación pública nacional

\# Invitación restringida

Fuente: Elaboración propia con base en los reportes de la unidad coordinadora del programa en los estados

diana para el tiempo de entrega fue de 469 días. Las obras ejecutadas a través de IR y LPN presentaron los lapsos de entrega más largos (medianas de 596 y 627 días, respectivamente), mientras que las unidades construidas con PC requirieron de sólo 246 días.

Como era de esperarse, los tiempos de construcción de las unidades de difícil acceso fueron considerablemente mayores. No obstante, las unidades de difícil acceso construidas con PC presentaron mejores tiempos de entrega que las unidades construidas por medio de LPN e IR.

Los 81 CSRD, por su parte, presentaron una distribución uniforme por lo que se refiere al tipo de acceso (cuadro II). En el rubro de modalidad de construcción predominó la LPN (46). De los CSRD construidos con PC (26), la mayoría (19) se construyó en Guerrero.

El gasto $/ \mathrm{m}^{2}$ de construcción fluctuó entre $\$ 543.4$ y $\$ 1$ 312.1. Como puede observarse en el cuadro II, la mediana de gasto $/ \mathrm{m}^{2}$ de construcción fue más baja cuando la obra se construyó con PC (\$634.9).

$\mathrm{El}$ gasto $/ \mathrm{m}^{2}$ según tipo de acceso fue muy similar para los tres grupos, con medianas de $\$ 802.7 / \mathrm{m}^{2}$ en zonas de acceso regular, $\$ 813.4 / \mathrm{m}^{2}$ en zonas de difícil 


\section{Cuadro II \\ GASTO/M² Y TIEMPOS DE CONSTRUCCIÓN EN CENTROS DE SALUD RURAL DISPERSOS, SEGÚN MODALIDAD de construcción y tipo de acceso. Programa de Apoyo a los Servicios de Salud para Población Abierta. Guerrero e Hidalgo, México, 2000}

\begin{tabular}{|c|c|c|c|c|c|c|c|c|c|c|c|c|}
\hline \multirow[b]{3}{*}{ Variable } & \multirow{2}{*}{\multicolumn{3}{|c|}{ Unidades }} & \multicolumn{9}{|c|}{ Gasto/m² y tiempos de construcción* } \\
\hline & & & & \multicolumn{3}{|c|}{ Mediana } & \multicolumn{3}{|c|}{ M ínimo } & \multicolumn{3}{|c|}{ Máximo } \\
\hline & Hidalgo & Guerrero & Total & Hidalgo & Guerrero & $\overline{\text { Total }}$ & Hidalgo & Guerrero & Total & Hidalgo & Guerrero & Total \\
\hline \multicolumn{13}{|c|}{ Gasto/m2 } \\
\hline \multicolumn{13}{|l|}{ Modalidad } \\
\hline$P C^{\ddagger}$ & 7 & 19 & 26 & 800.7 & 573.8 & 634.9 & 743.0 & 543.4 & 543.4 & 861.5 & 647.4 & 861.5 \\
\hline LPN $^{\S}$ & 2 & 44 & 46 & 901.1 & 878.2 & 879.2 & 772.9 & 809.1 & 772.9 & 1029.2 & 1312.1 & 1312.1 \\
\hline $\mathrm{IR}^{\#}$ & 3 & 6 & 9 & 979.5 & 964.3 & 846.2 & 846.2 & 705.2 & 705.2 & 1091.3 & 1127.3 & 1127.3 \\
\hline \multicolumn{13}{|l|}{ Acceso } \\
\hline Fácil & 3 & 26 & 29 & 811.2 & 814.1 & 813.8 & 772.9 & 543.4 & 543.4 & 853.4 & 1027.3 & 1027.3 \\
\hline Regular & 1 & 20 & 21 & 1029.2 & 791.4 & 802.7 & 1029.2 & 560.7 & 560.7 & 1029.2 & 1054.5 & 1054.5 \\
\hline Difícil & 8 & 23 & 31 & 860.3 & 797.0 & 813.4 & 743.0 & 560.7 & 560.7 & 1091.3 & 1312.1 & 1312.1 \\
\hline Global & 12 & 69 & 81 & 862.1 & 801.8 & 810.7 & 743.0 & 543.4 & 543.4 & 1091.3 & 1312.1 & 1312.1 \\
\hline
\end{tabular}

\begin{tabular}{|c|c|c|c|c|c|c|c|c|c|c|c|c|}
\hline \multicolumn{13}{|c|}{ ipo de co } \\
\hline$P C^{\ddagger}$ & 7 & 19 & 26 & 375 & 246 & 280 & 98 & 73 & 73 & 657 & 342 & 657 \\
\hline LPN $\S$ & 2 & 44 & 46 & 620 & 257 & 272 & 375 & 73 & 73 & 864 & 459 & 864 \\
\hline $\mathrm{IR}^{\#}$ & 3 & 6 & 9 & 646 & 175 & 332 & 488 & 77 & 77 & 835 & 325 & 835 \\
\hline
\end{tabular}

Acceso

\begin{tabular}{|c|c|c|c|c|c|c|c|c|c|c|c|c|}
\hline Fácil & 3 & 26 & 29 & 384 & 276 & 287 & 136 & 142 & 136 & 864 & 459 & 864 \\
\hline Regular & 1 & 20 & 21 & 375 & 237 & 244 & 375 & 77 & 77 & 375 & 398 & 398 \\
\hline Difícil & 8 & 23 & 31 & 534 & 221 & 302 & 98 & 73 & 73 & 835 & 398 & 835 \\
\hline Global & 12 & 69 & 81 & 483 & 246 & 282 & 98 & 73 & 73 & 864 & 459 & 864 \\
\hline
\end{tabular}

* Gasto/m² en pesos y tiempos de construcción en días

₹ Participación comunitaria

§ Licitación pública nacional

\# Invitación restringida

Fuente: Elaboración propia con base en los reportes de la unidad coordinadora del programa en los estados

acceso y de $\$ 813.8 / \mathrm{m}^{2}$ en zonas de fácil acceso. Guerrero presentó las medianas de gasto más bajas tanto por modalidad de construcción como por tipo de acceso.

Por lo que se refiere a los tiempos de conclusión de los CSRD, se obtuvieron medianas de 282 días, con una variación considerable entre Guerrero e Hidalgo (246 versus 483 días, respectivamente) (cuadro II). Es importante señalar que 33\% de los CSRD se concluyeron dentro de los plazos normados por PASSPA. Los mejores tiempos se presentaron en obras ejecutadas a través de LPN y PC (272 y 280 días de mediana, respectivamente). Guerrero tuvo también un mejor desempeño en ambos tipos de modalidad.

En términos generales, los CSRD ubicados en zonas de difícil acceso presentaron los tiempos de construcción más largos (mediana de 302 días). Sin embargo, los tiempos para estas unidades en Guerrero presentaron una mediana de 221 días, cifra menor incluso que la de los CSRD construidos en zonas de fácil acceso en ese mismo estado (276 días) y en Hidalgo (384 días). 
Finalmente, sólo cinco de los 29 CSRD construidos en localidades de fácil acceso se ajustaron al parámetro de 150 días, y sólo 14 de las 31 unidades de difícil acceso se ajustaron a la norma de 225 días de construcción.

Podemos así concluir que los gastos $/ \mathrm{m}^{2}$ más bajos se presentaron en los CSRD. Varias razones podrían explicar este hecho. En primer lugar, el tamaño de la unidad. Mientras mayores las unidades, mayores son las economías de escala. En segundo lugar, el acceso. Por definición, las UAS están ubicadas en zonas poco accesibles y con vías de comunicación muy pobres, hecho que encarece la construcción de obras.

Para complementar estos resultados y validar o rechazar la hipótesis de que las medianas del gasto/ $\mathrm{m}^{2}$ y tiempos de ejecución para cada una de las unidades objeto de este estudio son iguales, se realizaron dos pruebas estadísticas: Kruskal-Wallis y la prueba de U de Mann-Whitney. Las comparaciones se hicieron con relación a las distintas modalidades de construcción, tipo de acceso, tipo de unidad y entidad federativa.

\section{Cuadro III \\ GASTO/M² Y TIEMPOS DE CONSTRUCCIÓN para las unidades construidas por el Programa de Apoyo a los Servicios de Salud para Población Abierta, SEgún modalidad de CONSTRUCCIÓN. Global, México, 2000}

\begin{tabular}{|c|c|c|c|c|}
\hline \multirow{3}{*}{ Modalidad de construcción } & & \multicolumn{3}{|c|}{$\begin{array}{l}\text { Gasto/m² y tiempos } \\
\text { de construcción* }\end{array}$} \\
\hline & Unidades & Mediana & Mínimo & Máximo \\
\hline & Gasto/m² & & & \\
\hline$P C^{\ddagger}$ & 34 & 696.6 & 543.4 & 927.7 \\
\hline$\overline{\mathrm{LPN}}{ }^{\S}$ & 50 & 899.9 & 772.9 & 1312.1 \\
\hline $\mathrm{IR}^{\#}$ & 18 & 1020.3 & 705.2 & 1127.3 \\
\hline Global & 102 & 853.4 & 543.3 & 1312.1 \\
\hline
\end{tabular}

Tiempos de construcción

\begin{tabular}{lrrrr} 
PC & 34 & 272 & 73 & 657 \\
\hline LPN $^{\S}$ & 50 & 301 & 73 & 1001 \\
\hline IR $^{\#}$ & 18 & 464 & 77 & 928 \\
Global & 102 & 320 & 73 & 1001 \\
* Gasto/m² en pesos y tiempos de construcción en días & \\
₹ Participación comunitaria \\
§ Licitación pública nacional \\
\# Invitación restringida
\end{tabular}

Fuente: Elaboración propia con base en los reportes de la unidad coordinadora del programa en los estados

salud pública de méxico / vol.45, no.6, noviembre-diciembre de 2003
Como puede observarse en el cuadro III, la mayoría de las 102 unidades construidas se edificó a través de $\mathrm{LPN}$, con una mediana de gasto $/ \mathrm{m}^{2}$ de $\$ 899.9$, y gastos máximos y mínimos de $\$ 1312$ y $\$ 772.9 / \mathrm{m}^{2}$, respectivamente. Las 34 unidades construidas con $\mathrm{PC}$ presentaron una mediana de gasto $/ \mathrm{m}^{2}$ de $\$ 696.6$. Finalmente, las unidades construidas por IR presentaron una mediana de $\$ 1020.3 / \mathrm{m}^{2}$. De acuerdo con los resultados de la prueba de Kruskal-Wallis, estas diferencias son estadísticamente significativas $(p=0.000)$.

La prueba de U de Mann-Whitney demostró diferencias significativas en los gastos $/ \mathrm{m}^{2}$ favorables para los CSRD en relación con las UAS $(p=0.000)$. El mismo valor de $p$ se obtuvo al comparar las medianas de gasto de las distintas entidades federativas. En este rubro el estado de Guerrero fue el que presentó un desempeño más eficiente.

Por lo que se refiere al tiempo de construcción, las 102 unidades presentaron una mediana de 320 días, con una desviación estándar de 205 días (cuadro III). Por modalidad de construcción, las mejores cifras las presentaron las unidades construidas con PC (272 días). Las diferencias fueron estadísticamente significativas $(p=0.066)$.

Si el análisis lo basamos en el grado de accesibilidad de las unidades, observamos que el gasto $/ \mathrm{m}^{2}$ más bajo se presentó en las unidades construidas en zonas de fácil acceso (mediana de $\$ 818 / \mathrm{m}^{2}$ ) (cuadro IV). Las diferencias, sin embargo, al evaluarse con la prueba de Kruskal-Wallis, no resultaron estadísticamente significativas $(p=0.1281)$. Con la variable tiempo de construcción se obtuvieron resultados similares $(p=0.0863)$.

Por último, se desarrolló un análisis matemático de bootstrap (cuadro V) que permitió relacionar el gasto $/ \mathrm{m}^{2}$ con los tiempos de construcción, modalidad de edificación, tipo de unidad y la entidad federativa. Este modelo explica en $58 \%$ la variabilidad del gasto $/ \mathrm{m}^{2} \mathrm{y}$ nos permitió corroborar los hallazgos antes descritos. Es necesario destacar que se excluyó de este análisis la variable tipo de acceso dado que los resultados obtenidos mostraron que no era estadísticamente significativa.

A partir de los datos del cuadro $\mathrm{V} y$ tomando como unidad de comparación al gasto $/ \mathrm{m}^{2}$ de las UAS construidas con PC en zonas de fácil acceso del estado de Hidalgo, podemos observar que si se utilizan la LPN o la IR, el gasto $/ \mathrm{m}^{2}$ se incrementa en $\$ 267$ y $\$ 291$, respectivamente, en relación con el gasto $/ \mathrm{m}^{2}$ efectuado con PC. Si se comparan los distintos tipos de unidades, se observa que el gasto $/ \mathrm{m}^{2}$ de las UAS es $\$ 75 \mathrm{ma}-$ yor que el gasto $/ \mathrm{m}^{2}$ de los CSRD.

Otro aspecto importante que confirma lo comentado en párrafos anteriores, es el hecho de que la cons- 


\section{Cuadro IV \\ GASTO/M² Y TIEMPOS DE CONSTRUCCIÓN para las unidades construidas por el Programa de Apoyo a los Servicios de Salud para Población Abierta según tipo de acceso. Global, México, 2000}

\begin{tabular}{|c|c|c|c|c|}
\hline \multirow[b]{2}{*}{ Tipo de acceso } & \multirow[b]{2}{*}{ Unidades } & \multicolumn{3}{|c|}{$\begin{array}{l}\text { Gasto/m² y tiempos } \\
\text { de construcción* }\end{array}$} \\
\hline & & M ediana & Mínimo & Máximo \\
\hline \multicolumn{5}{|c|}{ Gasto/m² } \\
\hline Fácil & 31 & 818.1 & 543.4 & 1027.3 \\
\hline Regular & 25 & 831.3 & 560.7 & 1151.7 \\
\hline Difícil & 46 & 889.2 & 560.7 & 1312.1 \\
\hline Global & 102 & 853.4 & 543.3 & 1312.1 \\
\hline
\end{tabular}

Tiempos de construcción

\begin{tabular}{lrrrr} 
Fácil & 31 & 280 & 136 & 864 \\
\hline Regular & 25 & 263 & 77 & 775 \\
\hline Difícil & 46 & 378 & 73 & 1001 \\
Global & 102 & 320 & 73 & 1001
\end{tabular}

* Gasto $/ \mathrm{m}^{2}$ en pesos y tiempos de construcción en días

Fuente: Elaboración propia con base en los reportes de la unidad coordinadora del programa en los estados

trucción de unidades en Guerrero fue mucho más eficiente que en Hidalgo. De hecho, las cifras demuestran que en Guerrero se generó un ahorro de $\$ 242 / \mathrm{m}^{2}$ en comparación con las unidades construidas en Hidalgo.

Complementariamente al ejercicio anterior, se compararon los gastos de construcción de unidades en programas regulares de construcción de obra de la SSA, el IMSS y Pronasol, por un lado, y los gastos de construcción del PASSPA, por el otro. Para ello, primero se verificó que existiera similitud entre los tipos de unidades a comparar entre la SSA y el IMSS. Así, se observó que las UAS y los CSRD tienen su equivalente en el IMSS en la Unidad Médica Rural (UMR) y en la Unidad de Medicina Familiar (UMF) de uno y tres consultorios, respectivamente.

De acuerdo con la metodología antes citada, y previa actualización de los gastos $/ \mathrm{m}^{2}$ a términos reales de 1990, se observó que los gastos $/ \mathrm{m}^{2}$ de las UAS del programa regular de construcción de la SSA y de las

\begin{tabular}{|c|c|c|c|c|c|}
\hline \multicolumn{6}{|c|}{$\begin{array}{l}\text { Análisis matemático de ajuste a normal (bo otstrap) } \\
\text { Para el programa de obra del Programa de Apoyo } \\
\text { a los Servicios de Salud para Población Abierta. } \\
\text { Guerrero e Hidalgo, México, } 2000\end{array}$} \\
\hline Gasto/m² & $\begin{array}{l}\text { Coefi- } \\
\text { ciente }\end{array}$ & $\begin{array}{l}\text { Error } \\
\text { estándar }\end{array}$ & & IC 9 & $95 \%$ \\
\hline Tiempo de conclusión obras & -0.187 & 0.046 & 0.000 & -0.279 & -0.095 \\
\hline \multicolumn{6}{|l|}{ Modalidad } \\
\hline & 266.542 & 7.373 & 0.000 & 251.908 & 281.177 \\
\hline & 1.289 & 75.539 & 0.000 & 141.345 & \\
\hline
\end{tabular}

Tipo unidad

$\begin{array}{llllll}\text { CSRD }^{\S} & -75.792 & 38.149 & 0.050 & -151.518 & -0.066\end{array}$

Estado

\begin{tabular}{lrrrrr} 
Guerrero & -242.815 & 20.268 & 0.000 & -283.048 & -202.582 \\
\hline Constante & 941.663 & 33.728 & 0.000 & 874.714 & 1008.613
\end{tabular}

Unidad de comparación: U nidad Auxiliar de Salud construida por participación comunitaria en zonas de fácil acceso de Hidalgo

* Licitación pública nacional

₹ Invitación restringida

$\S$ Centro de salud rural disperso

Fuente: Elaboración propia con base en los reportes de la unidad coordinadora del programa en los estados

UMR ascendieron a $\$ 1230$, mientras que las UAS de Pronasol costaron en promedio $\$ 1$ 147. Por su parte, los gastos $/ \mathrm{m}^{2}$ de los CSRD y de los CSRC del programa regular de la SSA y de las UMF-1 y UMF-3 fueron de $\$ 1330$, mientras que los CSRC de Pronasol emplearon $\$ 930$ pesos $/ \mathrm{m}^{2}$.

Puede así concluirse que el gasto $/ \mathrm{m}^{2}$ de las UAS construidas por el PASSPA en Guerrero e Hidalgo (\$1 017) fue considerablemente menor que el de sus contrapartes de los programas regulares de la SSA y el IMSS ( $\$ 213$ menos por $\mathrm{m}^{2}$ ) e incluso que el de Pronasol. Si se considera como punto de comparación el gasto efectuado en las unidades construidas por PASSPA a través de PC los contrastes son todavía más significativos, ya que las UAS del PASSPA presentaron una mediana de $\$ 897.3 / \mathrm{m}^{2}$, lo que representa un ahorro de $17 \%$.

De igual forma, el gasto máximo $/ \mathrm{m}^{2}$ para los CSRD construidos a través de PC en los dos estados PASSPA (\$861.5) se ubicó por debajo del costo de sus equivalentes construidos por la SSA y el IMSS en sus programas regulares de obra civil. 


\section{Discusion}

Los resultados de esta investigación demuestran los beneficios presupuestales que puede generar la PC en la construcción de unidades de salud. Más específicamente, de estos resultados, se desprenden tres conclusiones:

- Que la PC en la construcción tanto de UAS como de CSRD es una modalidad que puede dar lugar a un gasto $/ \mathrm{m}^{2}$ considerablemente menor que otras modalidades de construcción (LPN e IR).

- Que aunque los tiempos de entrega no se apegaron a lo dispuesto por la norma, los tiempos de entrega de las unidades construidas con PC -sobre todo de las UAS- fueron mejores que los tiempos de entrega de las obras construidas con otras modalidades.

- Que la construcción de unidades básicas de salud bajo las condiciones generadas por el PASSPA -en particular la construcción con PC-puede mejorar el gasto $/ \mathrm{m}^{2}$ en relación con el de la construcción de obra de los programas regulares de construcción de la SSA, el IMSS y Pronasol.

Cabe también resaltar los resultados particularmente positivos de la construcción de obra con PC en el estado de Guerrero, en donde, por ejemplo, se reportaron gastos $/ \mathrm{m}^{2}$ y tiempos de entrega para los CSRD de difícil acceso menores a los de estas mismas unidades en zonas de fácil acceso de Hidalgo. Esto posiblemente se deba a la adopción por parte de Guerrero de experiencias previas que se generaron en Hidalgo, estado precursor de la construcción a través de PC, y a las adecuaciones realizadas en la programación y el desarrollo de las actividades después de la evaluación de medio camino efectuada en 1993.

Las diferencias en los gastos de construcción entre las distintas modalidades, en particular entre la PC, por un lado, y la IR y la LPN, por el otro, muy probablemente se deben a las ganancias que están incorporadas a la contabilidad de la construcción a través de proveedores privados. Sin embargo, los ahorros generados en algunos casos fueron tan importantes $\left(\$ 697 / \mathrm{m}^{2}\right.$ en las unidades construidas con PC versus $\$ 1020 / \mathrm{m}^{2}$ en las unidades construidas a través de IR) que bien podrían atribuirse también a algún otro mecanismo relacionado directamente con la participación comunitaria, como podría ser la adquisición de materiales a mejores precios o un manejo de los recursos financieros, materiales y humanos en general más eficiente.

La experiencia del PASSPA abre la posibilidad de considerar a la PC como una alternativa en otras acti- vidades regulares de los servicios de salud, como los programas de supervisión, los programas de mantenimiento o diversas actividades de salud pública.

Esta experiencia, además, muy posiblemente ayudó a fortalecer el espíritu comunitario y la disposición a contribuir al mejoramiento de las condiciones de vida de las comunidades que participaron en el programa. Sin embargo, las condiciones que ayudaron al éxito de la experiencia y el impacto que el programa de construcción con PC tuvo en las comunidades involucradas, serán motivo de discusiones más detalladas en otras publicaciones.

\section{Agradecimientos}

Los autores queremos hacer patente nuestro agradecimiento al maestro Ignacio Osuna Ramírez, por el apoyo brindado en la realización del análisis estadístico de los datos.

\section{Referencias}

1.Turabian JL.A puntes, esquemas y ejemplos de participación comunitaria en salud. Madrid, España: D ́az de Santos, 1991:1-11. 2. Fondo de las $\mathrm{N}$ aciones Unidas para la Infancia /W orld Health 0 rganization. Community involvement in primary health care.A study of the process of community motivation and continued participation. Ginebra:W HO, 1977.

3.W orld $\mathrm{H}$ ealth $\mathrm{O}$ rganization/Fondo de las $\mathrm{N}$ aciones Unidas para la Infancia. Primary health care. Report of the International Conference on Primary Health C are, Alma A ta, URSS. G inebra:W HO , 1978.

4.W orld Health $\mathrm{O}$ rganization. Formulating strategies for health for all by the year 2000. Ginebra:W HO, 1979 .

5. $O$ rganización Mundial de la Salud. Alma-Ata 1978. Atención Primaria a la Salud. Ginebra: O MS, 1978.

6. Zackus D, Lysack C. Revisiting community participation. Health Policy Plann 1998;13(1):1-12.

7. Klein R. N otes toward a theory of patient involvement. A commissioned paper to the community health center project. 0 ttawa: Canadian Public Health Association, 1973.

8. Dachler HP,W ilpert B. Conceptual dimensions and boundaries of participation in organizations:A critical evaluation. Adm Sci Q 1978;23:1-39.

9. Rifkin D, Muller F, Bichmann W. Primary health care: $0 \mathrm{n}$ measuring participation. Soc Sci Med 1988;26(9):931-940.

10. Freire P. Pedago gy of the oppressed. N ueva York: Herder \& Herder, 1970.

11. U galde A. Ideological dimensions of community participation in Latin American health programmes. Soc Sci Med 1985;21(1):41-53.

12. W orld Health $\mathrm{O}$ rganization Regional $\mathrm{O}$ ffice for Europe. European 0 bservatory on Health Care Systems, 2002. Copenhagen, D enmark: W HO, 2002.

13. Secretaría de Contraloría y D esarrollo Administrativo. Ley de adquisiciones y obras públicas. México, D F: SEC O DAM, 2 de octubre de 1996.

14. D rummond M, Stoddart G, Torrance G. Métodos para la evaluación económica de los programas de atención de la salud. Madrid, España: Díaz de Santos, 1991:7-129.

15. Secretaría de Salud. Modelo de atención a la salud para población abierta. México, DF: SSA, 1986. 